\title{
Error-Resilient MPEG-4 Video Communication over Error-Prone Wireless Networks
}

\author{
Tu-Chun Yin, Yi-Chin Huang, Meng-Hui Lin, Wen-Chin Chen \\ Communication and Multimedia Lab., \\ Department of Computer Science and Information Engineering, \\ National Taiwan University, Taipei, Taiwan \\ E-Mail: \{birding, yichin, slin,wcchen\}@cmlab.csie.ntu.edu.tw
}

\begin{abstract}
This paper presents an error-resilient MPEG-4 video communication system. The system comprises an error-resilient encoder, an adaptive error-resilient transcoder, an error-resilient decoder and the PLBP encodet/decoder. It has been evaluated under a configurable network error simulator. In addition, the system integrates the error resilience tools defined in MPEG-4 visual standard, namely Video Packet Structure, Header Extension Code (HEC) and NEWPRED, as well as three proposed novel error-resilient techniques, namely PrioritizedLevel Bitstream Protection (PLBP), Protected Data Partitioning (PDP) and Restorative Intra Adoption (RIA). In the decoder, three enror concealment techniques are implemented. They are temporal replacement, motion replacement, and predictive motion replacement. The decoder also implements an error detection mechanism that has high sensitivity to catch errors. Finally, the performance of the hybrid error-resilient system has been evaluated. The experimental results show that under the error-prone networks, acceptable video quality has been successfully retained without incurring much overhead.
\end{abstract}

\section{INTRODUCTION}

Wireless multimedia services will soon become a reality due to the advancement of telecommunication infrastructure, such as GPRS and 3G networks, and the improvement of network transmission quality. However, although technologies of wireless networks are getting much maturer, the essence of wireless networks does not change. Shading and multi-path effects of electromagnetic wave cause random and burst errors. Above network layer, applications still has to afford high end-to-end delay, packet loss, random and burst residual error, which cannot be resolved by channel coder below network layer. On the other hand, bandwidth is a scarce resource of wireless channel. Thus, low-bitrate transmission becomes an important issue for wireless applications. Therefore, video communication over ertoneous wireless channel requires the support of low bitrate and errorresilient capability. In this circumstance, MPEG-4 is an appropriate video coding scheme.

However, due to the vulnerability of the MPEG-4 video coding algorithms, two cases should be considered when a coded bitstream is hit by errors. In the first case, when an error is detected, the decoder has to discard all bits until next resynchronization point arrives. Lots of information is desolated and visual quatity is seriously damaged. For the other case, bits need not to be discarded. However, the decoder may get incorrect values and the visual quality is somewhat inevitably decreased.

Due to the features of MPEG-4 coding algorithms, the influence caused by any incorrectly decoded value or impairment of visual quality probably be propagated to other parts of the same VOP (spatial error propagation) or other VOPs (temporal error propagation). Consequently, to retain visual quality in erroneous circumstance, a video communication system has to reduce the range of the spatial error effect and stop the temporal error propagation.

\section{A Hybrid and Adaptive Error Resilient System}

As shown in figure 1 , the system consists of six modules, including Encoder, Network Enor Simulator, PLBP Encoder/Decoder, Transcoder and Decoder.

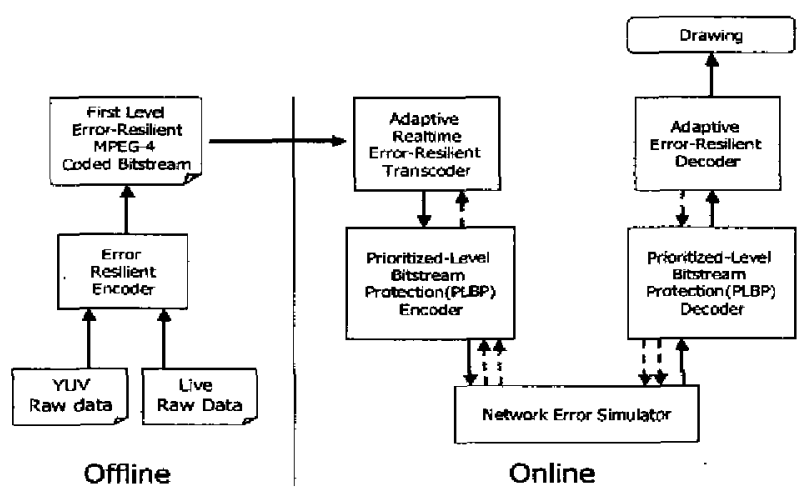

Figure 1. System Functional Block Overview

Encoder

Besides normal encoding functionalities, the encoder encodes motion areas in intra mode more frequently by adopting Adaptive Intra Refresh (AIR) mechanism. In a video presentation, human eyes tend to focus on high motion area. However, high motion area usually generates more bits than still area does. This implies high motion areas are more likely hit by error. Moreover, temporal predictive coding technique aggravates error propagation for high motion area. To solve the problem, AIR is adopted to help the decoder recover the corrupted motion area quickly and stop the error propagation. Motion analysis module and intra refresh decision module shall additionally be implemented in the encoder.

\section{PLBP Encoder/Decoder}

In order to prevent MPEG-4 bitstream from being destroyed by residual error, PLBP provides application-layer channel coding. The higher level of protection applying on data brings more channel-coding overhead. Thus, there exists a tradeoff between the level of protection and channel-coding overhead. On the other hand, it is observed that data which are more important are usually smaller. Thus, applying the same 
level of protection on the entire bitstream is not an efficient way. PLBP ensures different sections of a bitstream of different importance are protected by different channel coding algorithms with different data protection capability. As a whole, the bitstream gets optimal protection without incurring much overhead.

\section{Transcoder}

According to feedback messages from the decoder, the transcoder adaptively transcodes coded MPEG-4 bitstream in a real-time manner. Four error-resilient techniques are integrated, namely Video Packet Structure (VPS), Header Extension Code (HEC), Protected Data Partitioning (PDP) and New Predictive VOP Adoption (NPVA). NPVA comprises NEWPRED and Restorative Intra Adoption (RIA).

VPS reduces the range of spatial error propagation. Whenever the decoder loses synchronization with the bitstream due to some fatal decoding errors in one video packet (VP), it is able to resynchronize by the next VP in the same VOP. In order to realize resynchronization, spatial predictions of all macroblocks in a VP are bounded within the VP's boundary. In addition, macroblocks in a VP can only make temporal reference to the VP that is at the same location in the predicted VOP. Predictions outside a VP boundary shall be altered.

HEC makes each VP be decoded independently even without the information provided by the VOP header. If the VOP header is comupted by the transmission error, its information can be recovered by HEC.

PDP separates the motion and macroblock header information away to form the texture information. Motion information, which is smaller but more important than texture information, gets a higher-level protection under the operation of PLBP mechanism. If the texture information is lost, the decoder can conceal these errors by utilizing the motion information.

Errors introduced during transmission will lead to framestore mismatch between the transcoder and the decoder. NPVA helps the decoder to recover from temporal error propagations. It uses feedback messages to indicate which VP is erroneously decoded. In the transcoder, two transcoding strategies, namely Restorative Intra Adoption (RIA) and NEWPRED, will be applied altematively for each macroblocks that originally takes the lost video packet for temporal prediction. If a macroblock belongs to a portion of a high-motion area, RIA operates and the macroblock will be transcoded as intra-mode. Otherwise, NEWPRED operates and the transcoder re-encodes the macroblock by utilizing latest correctly decoded video packet at the same place of previous VOP for temporal prediction. In short, NPVA prevents temporal error propagation without inserting intra coded macroblocks and improves the visual quality in erroneous circumstances.

Decoder

As shown in Figure 2, the decoder implements an error detection mechanism that has high sensitivity to catch errors. When some error is detected, the decoder sends a message back to server. In addition, a lost area can be concealed by one of the following error concealment techniques, namely temporal replacement, motion replacement, and predictive motion replacement. The first one simply copies pels from the previous VOP. When the motion information is correctly decoded, the second scheme is utilized to conceal the error. If correct motion information is not available, the final technique utilizes the knowledge about spatial correlation to predict the most-likely motion information.

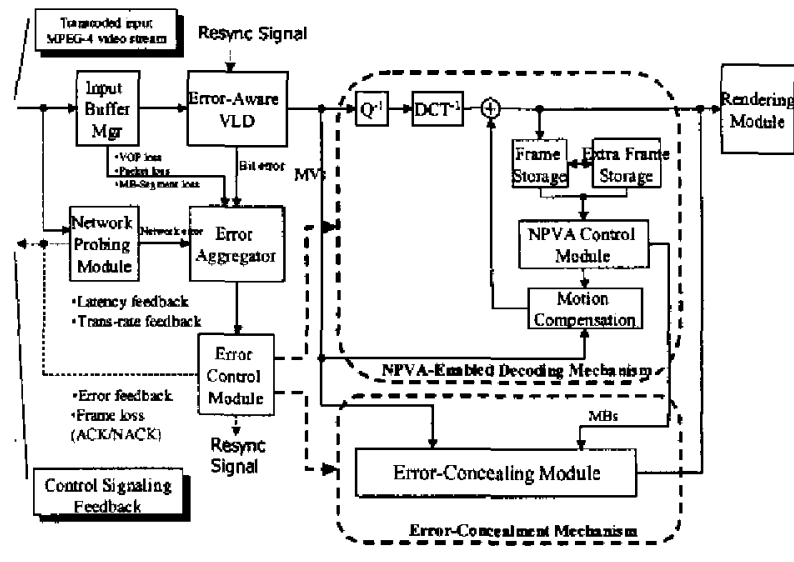

Figure 2. Architecture of Decoder

\section{Simulation Results}

Network error simulator generates the residual error that cannot be resolved in network layer. Three types of error are supported: packet loss, random error, and burst error. With appropriate configuration set on the network error simulator, performance of various error-resilient techniques can be evaluated over the simulated wireless channel.

\section{EXPERIMENTAL RESULTS AND CONCLUSIONS}

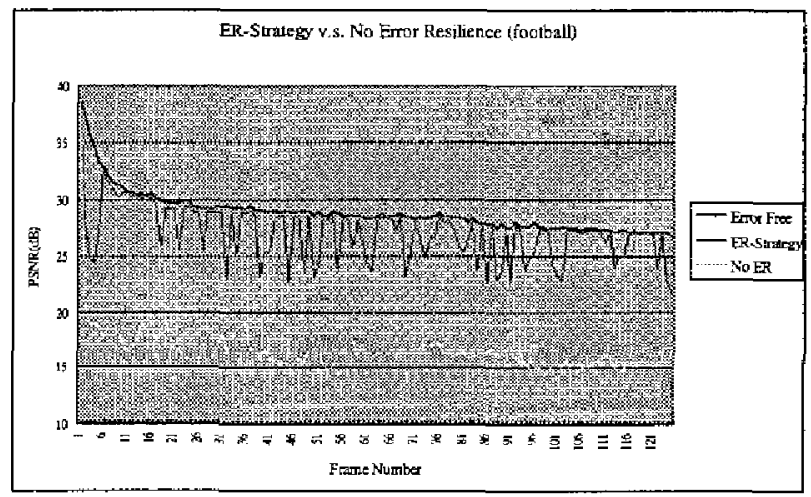

Figure 3. The PSNR results of "football." (I) The uppermost line error-free (2) the middle line: the error-resilient system (3) the third line: non-resilient and error-prone

The performance of the developed error resilient system was evaluated in the experiment. The test sequence "football" chosen for the simulation was encoded, transcoded and decoded in compliance with the MPEG-4 standard. The frame resolution, frame number, frame rate and bit rate were set to $352 \times 288,125$ frames, $10 \mathrm{fps}$ and $384 \mathrm{kbits} / \mathrm{s}$. The performance is tested through the network error simulator. BER and average error length of the error pattern was set to $1.35 \times 10^{-3}$ and 1.822 bits. Figure 3 presents the PSNR results of each frame. This result shows that in the best case, the error-resilient system improves PSNR from $15 \mathrm{~dB}$ to nearly $30 \mathrm{~dB}$ in average. Thus, the proposed system indeed retained acceptable video quality over error-prone wireless networks.

\section{REFERENCES}

[1] ISO/IEC 14496-2:2001(E), Part 2: Visual, "Information technologyCoding of audio visual objects"

[2] Ming-Ting Sun, Amy R. Reibman, "Compressed Video over Networks". Marcel Dekker, Inc 